\title{
Adaptive Information Providing System for R\&D Meeting Environments
}

\author{
Sang Keun Rhee, Jihye Lee, and Myon-Woong Park \\ Intelligence \& Interaction Research Center, \\ Korea Institute of Science and Technology, \\ 39-1 Hawolgok-dong, Seongbuk-gu, Seoul, Korea \\ \{greyrhee, bluesea, myon\} @kist.re.kr
}

\begin{abstract}
As extensive amount information is required within a $R \& D$ environment, an automated information system could save a considerable amount of time and efforts. There are currently many researches to overcome the information overload phenomenon, including information systems, recommender systems and adaptive hypermedia, and this paper presents an adaptive information providing system utilising some characteristics of these technologies to support R\&D and collaborative meeting environments. The system described in this paper aims to present the potential synergy between recommender systems and adaptive hypermedia systems.
\end{abstract}

Keywords: Adaptive Hypermedia, Context Awareness, Information System, Personalisation, Recommender System.

\section{Introduction}

Within a R\&D environment, a project is generally performed as a team-based collaborative work, and researchers and developers spend a significant amount of time and effort on searching, collecting and organising the necessary information. Not only the published papers but also informal documents created by individual researchers within $\mathrm{R} \& \mathrm{D}$ projects are valuable resources, and an automated system for managing, utilising, and recommending such resources can be beneficial to the $R \& D$ process. Moreover, as there usually are many meetings during a project, and the participants often require some additional information during a meeting, such an information system can also help the meeting processes.

Currently, there are various technologies developed to overcome the information overload phenomenon, including search engines and algorithms, recommender systems, adaptive hypermedia systems, and so on. These technologies play different roles depending on the applications, from decision making support to electronic learning aids. To support a R\&D process, we propose an automated information system utilising some characteristics of these technologies,

This paper presents an adaptive information providing system that helps organising various resources and provides automated recommendation of necessary data with context-awareness and personalisation, within a R\&D and meeting environment. 
First, the design principles of our system are discussed in four aspects - system role, resource management, context \& preference, and interface \& visualisation. Secondly, some related works are presented in this field including recommender systems, adaptive hypermedia, and R\&D support systems. Thirdly, the structure of our system is described with examples, and finally, it is concluded with discussions and some suggestions.

\section{Design Principles}

As mentioned in the previous section, the main role of our system is helping researchers and developers by providing the necessary information in the current context. However, there could be different methods and services that can achieve this purpose from various aspects, hence the four design principles of our system are discussed in this section.

\subsection{System Role}

The system is to be used in a R\&D environment, both in individual work processes and in meetings. The main role of this system is recommending necessary information based on the current context and individual user's characteristics, and the recommendation is to be generated automatically without explicit user queries. Regarding the environment, this system is to be used within a single project, in which the participants create or collect data and the resources are shared only among the team members. Furthermore, it provides some additional information to project managers including schedule, organisations, etc. Finally, this system can be implemented in a tangible meeting room for intelligent information services, which has been the fundamental motivation of this project within the Intelligent Responsive Space (IRS) project at KIST.

\subsection{Resource Management}

There might be various different kinds of information necessary in a R\&D environment. A researcher may be looking for specific technical documents or academic papers in a certain area, a project manager may seek a person with certain skills and backgrounds, a person may search for a certain project within his/her field, and so on. Therefore, it is necessary to clarify what is the information this system would provide to support a R\&D environment.

Though it might be often underestimated, the materials created or collected during a project - including informal or ill-structured documents - are in fact valuable information and it is not rare that people need some of those materials but have a hard time in finding them. Moreover, it becomes even harder when it comes to finding other person's material. Therefore, in our system design, the term resource is defined as the material created or collected by each participants of a certain project.

Instead of managing the resources as documents, the data objects are managed as units of fragments. A fragment is defined to be a reusable unit data object, and it can be either extracted from document resources or independently created. The basic unit data object is called an atomic fragment, and a compound fragment is a collection of 
these atomic fragments and/or other compound fragments. Each fragment has a specific set of metadata describing its characteristics. The main purpose of managing the resources as fragments is to provide adaptive documents - in other words, restructured and re-organised documents that only contain the contents suitable for the user's requirement.

\subsection{Context and Preference}

To generate a recommendation without an explicit user query, it is necessary to trigger an automated query generation process based on context information. In a R\&D environment, the environmental information is the overall project information, including the milestones and tasks, participants and their roles, meeting information both past meetings and future meeting plans - and so on. Instead of providing the same recommendation to every participant, it is also determined to design personal profiles reflecting individual characteristics and preferences so that the recommendation is both suitable to the current environmental status and personalised. More details on the context parameters can be found in Section 4.1 and also in [1].

\subsection{Visualisation and Interface}

Many recommender systems present recommendations as an ordered list [2][3]. Although this approach provides some benefits, it might be not the best technique for providing internal resources. Furthermore, during a meeting, it is not advisable to provide a long list of documents since the participants cannot spend too much time on reading the recommended data while others are in an active discussion. Therefore, it is decided to provide information in a single re-composed document form, whose contents is a collection of various fragments. Such document is to be generated each time the query results are given, and the contents are regarded as more important than the overall style since it is assumed that the users can understand the contents well enough even though the document is not in a perfectly well-structured form. Also, the main purpose of the generated document is to provide people with necessary information on the fly, not for personal study or authoring a formal document.

Compared to recommending a list of original documents, this approach can save some time for finding and understanding the required information. Furthermore, it is particularly useful for managing and re-using informal material created by individuals, and it also shows the benefit when the system is used within a meeting, where a user cannot spend a long time in reading his own material.

\section{Related Works}

Since our system shares some common characteristics with other works, it is worth mentioning some related works in this field before describing the details of our system.

\subsection{Recommender Systems}

In 1997, Resnick \& Varian defined recommender systems as computational systems in which 'people provide recommendations as inputs, which the system then aggregates and directs to appropriate recipients' [4]. Since then, the recommender 
systems have been advanced with various technologies [2], and applied in many different areas. In general, a recommender system 'offers recommendations to users based on their preferences' [3], and current recommender systems generally provide a list of recommended objects to support decision making.

Amazon or eBay is an example of successful implementation of a recommender system in e-commerce. In R\&D related domains, the Foxtrot system [5] is a good example which analyse the user's topics of interest and recommends academic papers in those topics.

\subsection{Adaptive Hypermedia Systems}

Adaptive hypermedia systems connects hypermedia and user modelling to adapt to the needs of users [6][7]. The two typical adaptation types are adaptive presentation and adaptive navigation support [7], and our system applied the former approach. Adaptive hypermedia systems have a strong connection with semantic web technologies and ontology, and are mostly advanced in e-learning area - SCARCE [8] and WHURLE [9] are good example applications.

In e-learning, adaptive hypermedia systems select, organise and present the contents based on user's background knowledge, goals, preference, etc., but usually the contents and their structures are clear and well-defined. In addition to the various existing issues in adaptive hypermedia, one great challenge of our system design was utilising the resources that are created by different individuals, without specific format.

IBM [10] and Nokia [11] developed this technology concentrating on device-based adaptive presentation, and UBiquoto [12] added user preference, location and time information to provide travel information.

\subsection{Systems Supporting Collaborative Work Environment}

Apart from information systems, there are active researches in Human-Computer Interaction area to support collaborative work environments. Some of them also have information services as well as intelligent and interactive interfaces.

Access Grid [13] is a well known technology to support video conferencing, and has been implemented in various forms of creative and academic collaborations around the world. MIT project Oxygen [14] is another successful example of collaborative work support system,

\section{The System}

The main functionality of our system is providing adaptive information to researchers and developers, and it shares some common features with recommender systems and with adaptive hypermedia systems. The prototype system presented in this paper provides the necessary data resource during a R\&D process and in meetings without explicit queries from users, by searching and selecting the relevant data based on the user profiles and other context information. The query results are then re-structured and organised to form a single document, yet it lacks navigation support unlike other adaptive web information systems (AWIS) [6][7]. This system has slight emphasis on its usage within meetings, but it can also be applied for general R\&D processes. The system overview is shown in Fig. 1. 


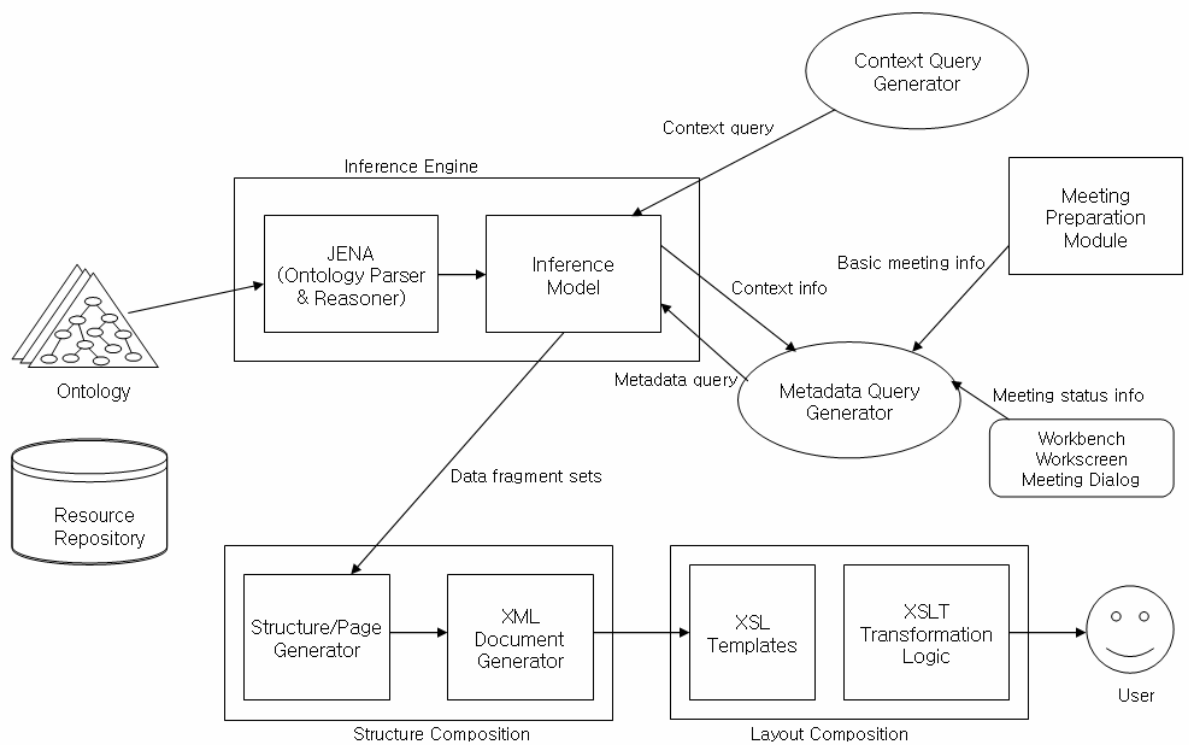

Fig. 1. The system structure

\subsection{The Information Model for Adaptive Composition}

To organise the data objects and provide the right information by context-awareness, it is firstly required to model the various elements and properties of a $R \& D$ environment. Considering the design principles of our system, the Information Model for Adaptive Composition (IMAC) [1] was suggested which defines the fundamental structure and base schema of the data resources, people, and environment within the R\&D domain (Fig. 2 shows the IMAC overview). Our system utilised the IMAC in part, and each domain and its elements are structured and represented in ontological form.

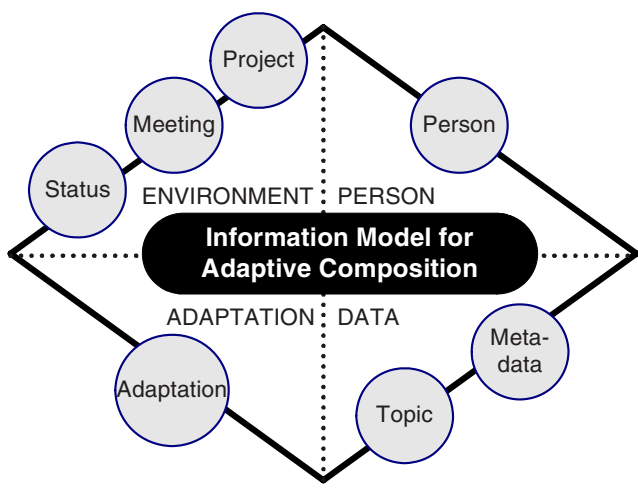

Fig. 2. The IMAC overview. More details of the IMAC can be found in [1] 
Resources. The resources (fragments) are classified and stored within the Topic Domain. The Topic domain is built based on the DMoz Open Directory Project (DMoz ODP) [15] structure, with some manual modification. The directory categories and subcategories are defined as classes, and the relations between categories are defined as object properties. In addition to this classification, the metadata based on the Metadata Domain is assigned to each fragment to specify its properties and relations with other fragments.

Contexts. To generate the correct query at a given context, the environmental information is also stored in the ontology. The Project Domain the overall project information including task hierarchies, related topics, participants, and so forth. The Meeting Domain analyses the meeting information and their agenda, and finally the current situation is computed utilizing the Status Domain. In addition to these environmental information, personal tasks, roles, preferences, etc. for individuals are stored in Person Domain to generate personalised results.

Ontology. The domain and elements of the IMAC is represented as an ontology in OWL DL using Protégé 3.1.1[16]. (Fig. 3.)

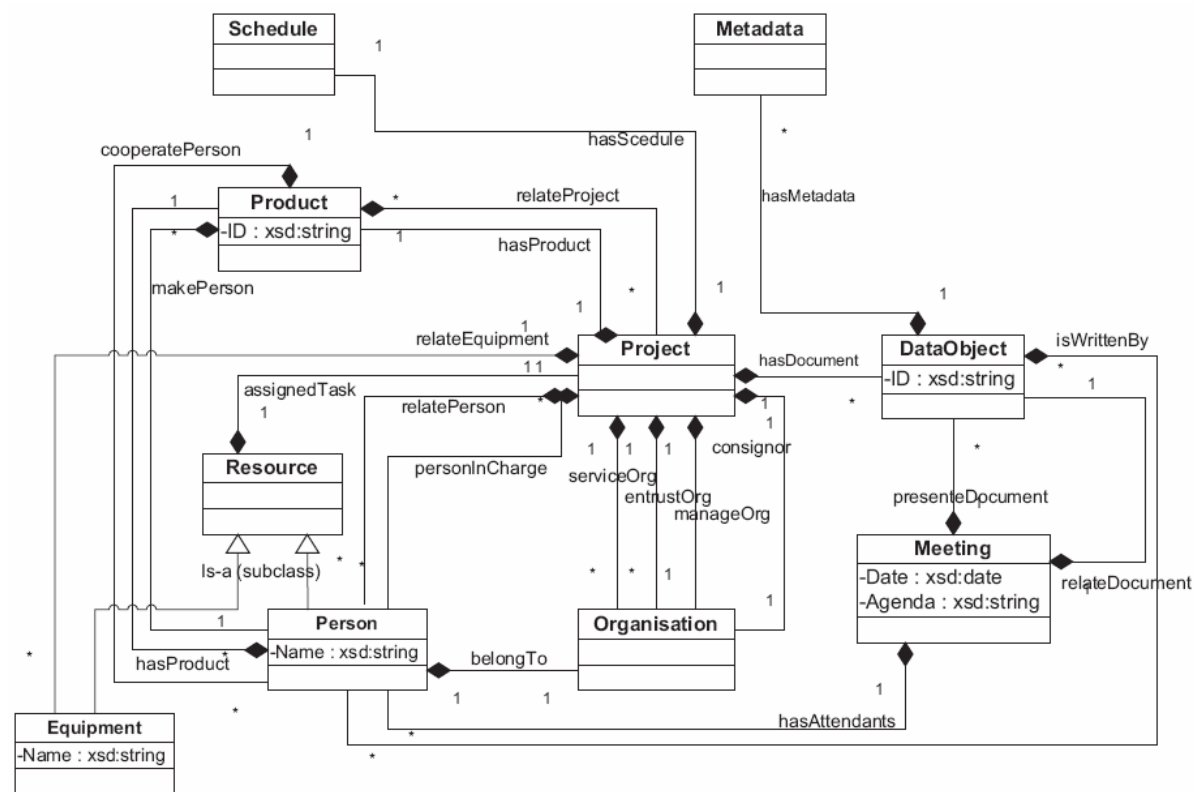

Fig. 3. The ontology structure

\subsection{Selection and Composition}

The first step for query generation is selecting the necessary topics for the current context. The system sees the topic structure as a labelled directed graph, with each topic as a node and each relation between topics as an edge. Each edge is labelled 
with one of the four relation types - is-a(super topic), narrow(subtopic), related, and symbolic. For each relation type, a numeric value is assigned representing the distance between two topics, and the value is set according to several tests.

To find the topic(s) required within the current context, the project, tasks and related topic information is extracted from the Project and Person domains. In addition, personal task, role, and preferences are analysed for personalization, and the meeting agenda and related tasks from the Meeting domain are also included in case this system is used within a meeting. Based on this context information, an ordered list of the related topics is generated by navigating the topic graph.

Once the topics are selected, the system then gathers the appropriate fragments within the selected topics. Concerning the user's knowledge and language level, task, the relation value (distance) between the selected topics, and the relations between each fragments, the final target fragments are selected and these are structured into an XML document format. This XML document is then parsed to generate a HTML page via XSL/XSLT. Fig. 4 presents an example result page, which may seem to be an ordinary single document but is actually a structured set of around 40 fragments.

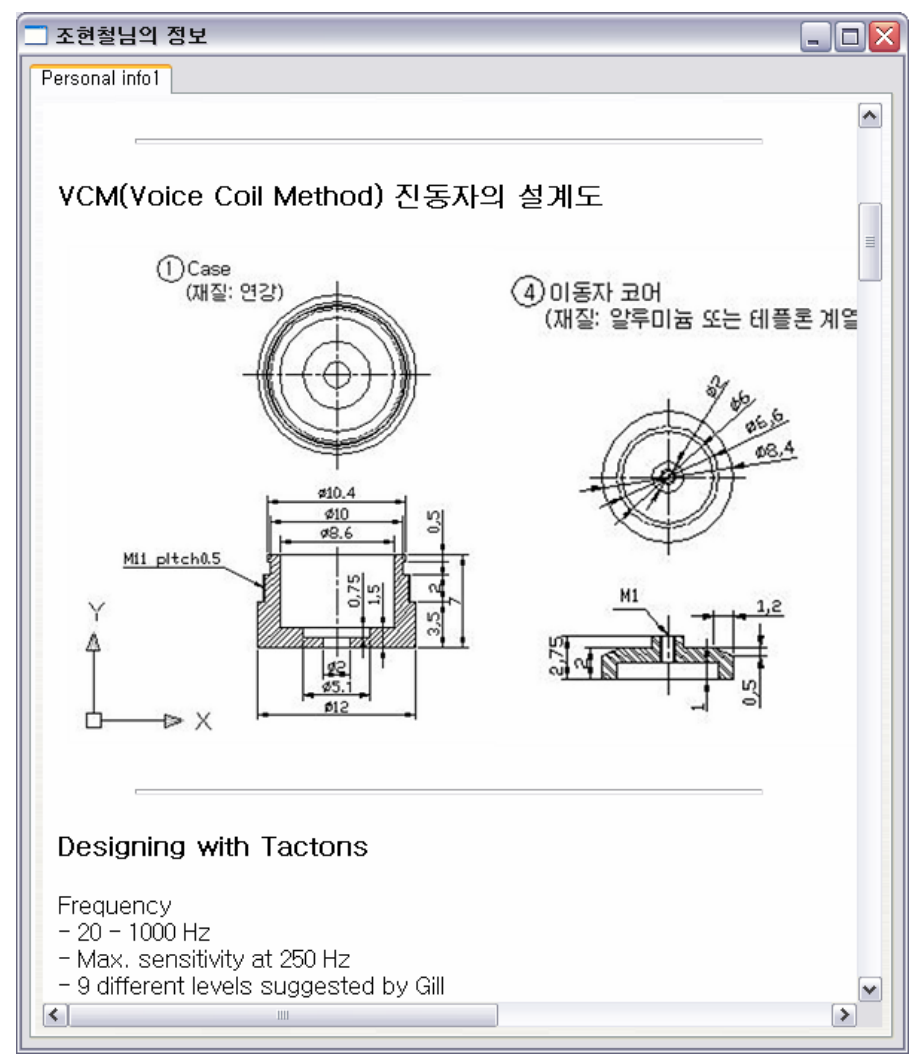

Fig. 3. An example of a generated document. In this example, titles, a picture, and texts are all separate fragments, and the horizontal lines indicate groups of fragments with related contents. 


\section{Discussion}

The system presented in this paper is a prototype that was designed and developed to actively provide personalised information in an $R \& D$ environment. The main objective of this paper is to present and discuss the possibility of utilising the methods and techniques used in recommender systems and adaptive hypermedia systems. The adaptive composition method used here enables users to receive the core contents that can be read and understood reasonably quickly but there are still several issues and much work is left to be done in the future.

Currently, the system requires too much users' effort. The users, including the project manager and/or system administrator, need to set various context information and user profiles manually. Also, when updating a new data fragment, its metadata needs to be assigned manually. In addition, at the early stage of a project, this system can hardly be of any help since there are few resources to provide, apart from initialisation of context information and preferences. This problem, known as the cold-start problem, appears to be much severe than other recommender systems. Regarding the result visualisation, it needs further testing and survey to answer the question whether providing the resource as a restructured set of fragments form is actually better than providing the original document lists.

This system can be improved by including some additional functionalities, and those would be the next step in our research. First, automating the fragments and their metadata generation process would greatly reduce users' effort, and it would enable the system to crawling and gathering external resources. The access level to each fragment can also be set for security and privacy issues. Second, automatic profile update based on both implicit and explicit feedback would improve the precision of the result. Third, this system can be combined with a generic project management or scheduling system, which would help assigning some of the context information including the Project and Meeting domains. Finally, more precise meeting information could be gathered and analysed in the intelligent meeting room environment, which can be used to automatically generate meeting minutes and better recommendations.

To sum up, this paper shows the directions and possibility of a new method in information systems, but the users' effort is not worth the reward at the current stage. This problem can be solved by applying various techniques discussed above. In addition, there are various other ways of applying and improving this system design developing a more generic ontology that can be applied for other related domain applications, applying more individual characteristics such as learning style [17] or other cognitive properties [18] for personalisation, adapting the multi-agent system structure, and so on.

Acknowledgments. This work was supported by the Korea Institute of Science and Technology (KIST) with the project IRS (Intelligent Responsive Space), which is a part of TSI (Tangible Space Initiative) project. 


\section{References}

1. Rhee, S.K., et al.: Information Modelling for Adaptive Composition in Collaborative Work Environment. Enformatika 14, 151-156 (2006)

2. Burke, R.: Hybrid Recommender Systems: Survey and Experiments. User Modeling and User-Adapted Interaction 12, 331-370 (2002)

3. Terveen, L.G., Hill, W.: Beyond Recommender Systems: Helping People Help Each Other. In: Carroll, J. (ed.) HCI in the New Millennium, Addison Wesley, New York (2001)

4. Resnick, P., Varian, H.R.: Recommender systems. Communications of the ACM 40(3), 56-58 (1997)

5. Middleton, S.E., Shadbolt, N.R., De Roure, D.C.: Ontological User Profiling in Recommender Systems. ACM Transactions on Information Systems 22(1), 54-58 (2004)

6. Brusilovsky, P.: Methods and Techniques of Adaptive Hypermedia. User Modeling and User Modeling and User-Adapted Interaction 6(2-3), 87-110 (1996)

7. Brusilovsky, P.: Adaptive Hypermedia, User Modeling and User-Adapted Interaction 11, 87-110. Springer, Netherlands (2001)

8. Iksal, S., Garlatti, S.: Adaptive Web Information Systems: Architecture and Methodology for Reusing Content. In: Proc. 1st International Workshop on Engineering the Adaptive Web, Eindhoven, the Netherlands (2004)

9. Moore, A., Brailsford, T.J., Stewart, C.D.: Personally tailored teaching in WHURLE using conditional transclusion. In: Proceedings of the 12th ACM conference on Hypertext and Hypermedia, 163-164 (2001)

10. Britton, K.H.: Transcoding: Extending e-business to new environments, IBM Systems Journal, 40(1) (2001)

11. Metso, M., et al.: Mobile Multimedia Services - Content Adaptation. In: 3rd International Conference on Information, Communications and Signal Processing, Singapore (2001)

12. Cena, F., et al.: Integrating Heterogeneous Adaptation Techniques to Build a Flexible and Usable Mobile Tourist Guide. AI Communications 19(4), 369-384 (2006)

13. Access Grid Project Homepage, http://www.accessgrid.org/

14. MIT Project Oxygen Homepage, http://www.oxygen.lcs.mit.edu/

15. DMoz Open Directory Project, http://dmoz.org/

16. The Protégé Ontology Editor and Knowledge Acquisition System, http://protege.stanford.edu/

17. Grigoriadou, M., Papanikolaou, K., Kornilakis, H., Magoulas, G.: INSPIRE: An Intelligent System for Personalized Instruction in a Remote Environment. In: Proceedings of 3rd Workshop on Adaptive Hypertext and Hypermedia, Sonthofen, Germany, 13-24 (2001)

18. Tarpin-Bernard, F., Habieb-Mammer, H.: Modeling Elementary Cognitive Abilities for Adaptive Hypermedia Presentation. User Modeling and User-Adapted Interaction 15(5), 459-495 (2005) 